\title{
Novel efficient THz undulator using a laser-driven wire
}

\author{
Kazuhisa Nakajima \\ Light: Science \& Applications (2017) 6, e17063; doi:10.1038/Isa.2017.63; published online 19 May 2017
}

Terahertz $\left(\mathrm{THz}=10^{12}\right.$ cycles per second, or $T$-ray) radiation sources are indispensable for modern materials science, chemical and biomedical imaging and sensing, as well as for a broad range of applications in diagnostic systems and advanced telecommunications ${ }^{1,2}$. The electromagnetic spectrum of the $\mathrm{THz}$ radiation is loosely characterized by the frequency range of $0.1-10 \mathrm{THz}$, or wavelengths between $30 \mu \mathrm{m}$ and $3 \mathrm{~mm}$. In particular, a low photon energy of $4.2 \mathrm{meV}$ or $48 \mathrm{~K}$ at $1 \mathrm{THz}$, which is less than the thermal energy at room temperature, allows precise measurements with a simplified setup and very high signal-to-noise ratio in time-domain pulsed $\mathrm{THz}$ spectroscopy ${ }^{2}$. Since the spectral region is located on the boundary between electronics and photonics, the diversity of the $\mathrm{THz}$ sources relies on either frequency up-conversion of electronic sources, such as solid-state photo-switches and radio-frequency (RF) electron-beam accelerators ${ }^{3}$, or frequency down-conversion of optical sources, such as optical rectification ${ }^{2,3}$ in nonlinear optical processes and laser-gas interaction induced by twocolor lasers ${ }^{3}$. Both technologies, however, exploit ultrashort pulse lasers as a driver. In a recent publication, Tian et al. ${ }^{4}$ at Shanghai Institute of Optics and Fine Mechanics (SIOM) and Nankai University developed a new $\mathrm{THz}$ radiation source using a laser-driven wire that generates a femtosecond electron bunch and helical undulator fields to emit $\mathrm{THz}$ synchrotron radiation with high efficiency.

Relativistic electron beams render ubiquitous sources of broadband $\mathrm{THz}$ radiation through various physical mechanisms; for instance, synchrotron radiation in bending magnetic fields and undulator fields, transition radiation, Cherenkov radiation, diffraction radiation and Smith-Purcell radiation. When the dimensions of an electron bunch are smaller than the $\mathrm{THz}$ wavelength, spontaneous emission of the $\mathrm{THz}$ radiation from individual electrons builds up in a coherent manner. This produces so-called coherent or super-radiant radiation, implying that all electrons inside the bunch radiate electromagnetic fields in the same phase, which interfere constructively, and the intensity increases with the square of the number of electrons.

Coherent transition radiation (CTR), characterized by a broadband spectrum and high-energy single-cycle pulse, is generated by the interaction of ultra-relativistic electron bunches accelerated from an $\mathrm{RF}$ linear accelerator with a metal foil. Such $\mathrm{THz}$ sources have been operated parasitically at fourth-generation X-ray light sources. For example, relativistic electron bunches with energy of $14 \mathrm{GeV}$, charge of $350 \mathrm{pC}$ and duration of $50 \mathrm{fs}(15 \mu \mathrm{m}$, bunch length) generate CTR with $\sim 200 \mu \mathrm{J}$ per pulse at $2.5 \mathrm{THz}$ through a $10-\mu \mathrm{m}$-thick Be foil at the Linac Coherent Light Source (LCLS) 5 .

Meanwhile, relativistic electron beams with relativistic factor $\gamma\left(=E_{\mathrm{e}} / m c^{2}\right.$ with electron energy $E_{\mathrm{e}}$ and rest energy $\left.m c^{2}=511 \mathrm{keV}\right)$ delivered from RF accelerators can generate synchrotron radiation, the wavelength of which is Doppler-shifted by a factor $\sim 1 / 2 \gamma^{2}$ in the passage through an alternating magnetic field $B_{u}$ of the undulator with period $\lambda_{u}$. Under the resonance condition that radiation emitted in one undulator period overtakes the electron and interferes constructively with radiation emitted in the next undulator period, that is, the radiation wavelength $\lambda_{r}=\lambda_{u}\left(1+K^{2} / 2\right) / 2 \gamma^{2}$ with the parameter $K=e B_{u} \lambda_{u} / 2 \pi m c^{2} \approx 0.934 B_{u}[\mathrm{~T}] \lambda_{u}[\mathrm{~cm}]$, the radiation at the end of the undulator periods $N_{u}$ has a spectral bandwidth $\Delta \omega / \omega=1 / N_{u}$. In a low-gain free electron laser (FEL), where the undulator is installed inside an optical cavity, the radiation via the spontaneous emission process from the electron beam is stored and amplified throughout the stimulated emission process inside the cavity, which is analogous to a conventional laser. As a consequence of the interaction between electrons and radiation, the electron beam undergoes the energy modulation, resulting in spatial modulation (microbunching) at the radiation wavelength. This FEL process transforms incoherent emissions from individual electrons with initially random phases into their coherent emissions with the same phase. As developed in modern $\mathrm{X}$-ray FELs, a high-gain mechanism exploiting high-peak current electron beams, referred to as a self-amplified spontaneous emission FEL, enables saturation to be reached in a single pass through the undulator. After compression to microscopic length scales in an RF accelerator, ultrashort electron bunches are injected into a long undulator, and the spontaneous emission in the first periods of the undulator is amplified through the energy modulation and microbunching processes. For example, a nine-pole electromagnetic undulator installed at the FEL in Hamburg (FLASH) generated pulse energy of up to $100 \mu \mathrm{J}$ at $1 \mathrm{THz}$ with a spectral bandwidth of $10 \%$ from $500 \mathrm{MeV}$ electron bunches with $0.6 \mathrm{nC}$ charge ${ }^{3,6}$.

While relativistic electron beam-driven $\mathrm{THz}$ sources, such as CTR and the $\mathrm{THz}$ undulator, are capable of generating high-energy $\mathrm{THz}$ pulses with $>100 \mu \mathrm{J}$ only at large-scale accelerator facilities, laserdriven $\mathrm{THz}$ sources, such as semiconductor photoswitches and nonlinear optical crystals, are suited to small-scale ubiquitous applications requiring limited pulse energies of the order of $1 \mu \mathrm{J}$, which is 


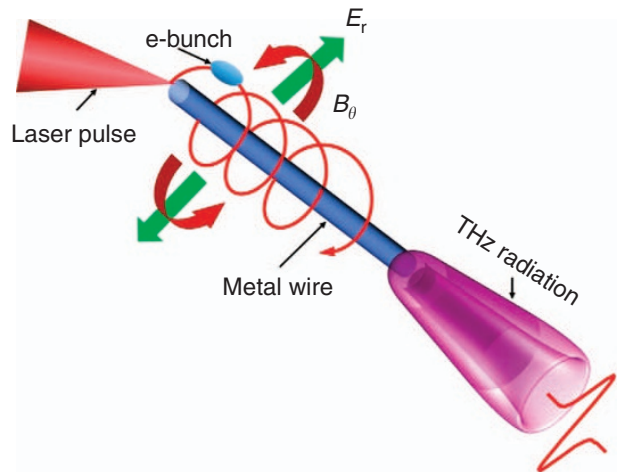

Figure 1 A schematic view of the laser-driven-wire-guided undulator (LWGU) developed at SIOM. A femtosecond laser pulse irradiating a metal wire emits an energetic electron bunch. Concurrently, a highly positive transient current excites an outward-pointing radial electric field $\left(E_{r}\right)$ and azimuthal magnetic field $\left(B_{\theta}\right)$ along the wire. The laser-generated fast electrons undergo helical betatron motion due to strong Lorentz forces and emit amplified $\mathrm{THz}$ radiation.

restricted due to dielectric breakdown. The new THz emitter, named laser-driven-wire-guided undulator (LWGU), developed at SIOM, combines capabilities of high-energy pulse generation pertaining to the undulator scheme and an extremely simplified miniature device comprising a millimeter-scale metal wire that functions as an energetic electron-beam source, helical undulator, waveguide and antenna of $\mathrm{THz}$ radiation. In the LWGU experiment, an electron bunch having a typical energy of $100 \mathrm{keV}$ and charge of at least $300 \mathrm{pC}$ was created on the surface of a metal wire with $50-90 \mu \mathrm{m}$ diameter while being irradiated by a $30-\mathrm{fs}, 3-\mathrm{mJ}$ laser operated at a repetition rate of $1 \mathrm{kHz}$. Concurrently, an intense transient current induced in the wire excited an outward-pointing radial electric field of the order of $\sim 0.3 \mathrm{GV} \mathrm{m}^{-1}$ and an azimuthal magnetic field $\sim 10 \mathrm{~T}$ in a decay time of $\sim 2 \mathrm{ps}$, owing to a positive-charge pulse propagating along the wire nearly at the speed of light. The laser-generated fast electrons undergo helical betatron motion due to strong Lorentz forces along the wire and emit synchrotron radiation (Figure 1). The $\mathrm{THz}$ radiation was characterized with a single-shot pump probe technique using the electro-optic sampling method to measure a temporal $\mathrm{THz}$ pulse waveform and Fourier-transformed spectrum. The spectral measurements show a tuneable peak frequency from 0.12 to $0.35 \mathrm{THz}$ with the bandwidth $0.09-0.17 \mathrm{THz}$ when varying the wire diameter from 90 to $50 \mu \mathrm{m}$. The angular-distribution measurement shows a typical $\mathrm{THz}$ emission concentrated within a narrow cone between $10^{\circ}$ and $30^{\circ}$, while the $\mathrm{THz}$ wave has mainly a radial polarization similar to the direction of the induced electric field. The total energy of $\mathrm{THz}$ emission was $\sim 28 \mu \mathrm{J}$ in the $0.1-0.3 \mathrm{THz}$ band for a $10-\mathrm{cm}$ long wire with a $50-\mu \mathrm{m}$ diameter driven by a 3-mJ laser pulse, which means a conversion efficiency of $\sim 1 \%$. It is noted that the energy-conversion efficiency of the LWGU is much higher than those of large-scale accelerator-based $\mathrm{THz}$ sources, such as $0.004 \%$ for CTR at LCLS and $0.03 \%$ for $\mathrm{THz}$ FEL at FLASH. The overall performance of the LWGU has confirmed the dominant properties of FELs, namely, frequency-tunability, narrow bandwidth, indication of gain saturation depending on the undulator length and 1\%-level energy-conversion efficiency.

The new femtosecond laser-driven $\mathrm{THz}$ radiation source, LWGU, may bring many beneficial properties in power, efficiency and stability as well as in operational and structural simplicity to currently prevailing $\mathrm{THz}$ devices for a wide range of applications. To understand the underlying physics of $\mathrm{THz}$ emission by the LWGU and further improve the performance of the $\mathrm{THz}$ source, it will be necessary to implement $a b$ initio analysis of the physical processes for $\mathrm{THz}$ radiation, for instance, laser-solid interactions, free electron generation, transient current generation, self-excitation of electromagnetic fields, and $\mathrm{THz}$ wave generation and guiding, in addition to phenomenological modeling. Such comprehensive investigations on the $\mathrm{THz}$ radiation sources may evoke not only technical innovations but also new insights into modern plasmonics research on surface plasmon-polaritons.

\section{CONFLICT OF INTEREST}

The author declares no conflict of interest.

1 Ferguson B, Zhang XC. Materials for terahertz science and technology. Nat Mater 2002; 1: 26-33.

2 Ulbricht R, Hendry E, Shan J, Heinz TF, Bonn M. Carrier dynamics in semiconductors studied with time-resolved terahertz spectroscopy. Rev Mod Phys 2011; 83: 543-586.

3 Stojanovic N, Drescher M. Accelerator- and laser-based sources of high-field terahertz pulses. J Phys B At Mol Opt Phys 2013; 46: 192001.

4 Tian Y, Liu JS, Bai YF, Zhou SY, Sun HY et al. Femtosecond-laser-driven wire-guided helical undulator for intense terahertz radiation. Nat Photon 2017; 11: 242-246.

5 Wu ZR, Fisher AS, Goodfellow J, Fuchs M, Daranciang D et al. Intense terahertz pulses from SLAC electron beams using coherent transition radiation. Rev Sci Instrum 2013; 84: 022701

6 Gensch M, Bittner L, Chesnov A, Delsim-Hashemi H, Drescher M et al. New infrared undulator beamline at FLASH. Infrared Phys Technol 2008; 51: 423-425.

(c) $($ ) $\ominus$ This work is licensed under a Creative Commons AttributionBY NC ND NonCommercial-NoDerivs 4.0 International License. The images or other third party material in this article are included in the article's Creative Commons license, unless indicated otherwise in the credit line; if the material is not included under the Creative Commons license, users will need to obtain permission from the license holder to reproduce the material. To view a copy of this license, visit http:// creativecommons.org/licenses/by-nc-nd/4.0/

(C) The Author(s) 2017 